significant. ZBTB2, ZBTB17, ZNF131 did not have, all genes were compared to HIV negative subjects. No significant changes were observed in pro-inflammatory cytokines levels, however, in treated patients, IL-1 $\beta$, TNF- $\alpha$, IL-6, IL-10 and IL-12 showed lower levels compared with naïve patients.

Conclusion We showed an increase gene expression of some ZBTBs in HIV+ patients compared with healthy donors, these genes have been related to the inflammatory cytokines suppression; we found that this difference was higher in treated patients compared to naïve patients, this could be related to a decrease in TNF- $\alpha$, IL-6, IL-10 and IL-12 systemic levels.

Disclosure No significant relationships.

\section{P172 ASSOCIATION BETWEEN SHORT-CHAIN FATTY ACIDS PRODUCING BACTERIA AND CD4 T CELLS RECOVERY IN HIV POSITIVE PATIENTS}

${ }^{1}$ Mariana Ruiz Briseño*, 'Sarah Ratkovich-Gonzalez, ${ }^{1}$ Monserrat Alvarez-Zavala, ${ }^{1}$ Alejandra Vega Magaña, ${ }^{2}$ Luz Gonzalez-Hernandez, 'Moises Ramos Solano, ${ }^{2}$ Jaime AndradeVillanueva. 'Universidad de Guadalajara, Guadalajara, Mexico; ${ }^{2}$ Hospital Civil Fray Antonio Alcalde, Unidad De VIH, Guadalajara, Mexico

\subsection{6/sextrans-2019-sti.331}

Background Antiretroviral Therapy (ART) inhibits HIV replication, allowing immune reconstitution; however, some patients have an insufficient reconstitution, resulting in high levels of immune activation, microbial translocation, inflammation and intestinal dysbiosis. Since microbiota and its metabolites, such as Short-Chain Fatty Acids (SCFA) are linked to immune status, alteration of one or both could be related to poor $\mathrm{T}$ cells reconstitution.

Methods HIV+ patients with ART, grouped as immunologic responders, IRs: $>350$ cells $(n=18)$, immunologic non-responders, INRs: $<350$ cells $(n=17)$ and, healthy controls $(n=$ 14) were recruited. Absolute quantification of Firmicutes, Bacteroidetes, Actinobacteria, Proteobacteria, Bifidobacterium, Clostridium leptum, Clostridium coccoides, Lactobacillus and Faecalibacterium prausnitzii were measured in stool by qPCR. Levels of butyrate, propionate and acetate were quantified in stool by HPLC. Absolute CD4 nadir count, CD4/CD8 proportion and co-expression of HLA-DR/CD38 were determined by flow cytometry. Comparisons between groups were performed with Kruskal-Wallis test.

Results No differences were found in the four main phyla. Regarding probiotics, there were no differences in Lactobacillus and C. coccoides; however, IRs have less copies of C. leptum and $F$. prausnitzii in comparison to the other groups. In contrast, INRs presented similar amounts of probiotics as the healthy subjects. No differences were found in SCFA levels, except for acetate which was increased in IRs. Concerning the immune status, there was no difference in CD4 nadir; however, IRs had greater variation in this count and, significantly higher CD4/CD8 proportion. Whereas, co-expression of CD4 + HLA-DR+CD38+ was decreased in INRs in comparison with IRs.
Conclusion Immune status from HIV + subjects directly affects microbiota composition, systemic activation and inflammation. The enrichment of some probiotics, particularly SCFA-producing bacteria, is related to deficient immune reconstitution; however, other gut bacteria could be compensating this decrease. Further studies are necessary to understand how the microbiota and their metabolic products are related to CD4 T cells recovery.

Disclosure No significant relationships.

\section{P173 INVESTIGATING VARICELLA-ZOSTER VIRUS-SPECIFIC T CELLS THROUGH THE LENSES OF HIV}

${ }^{1}$ Carolina Moreira*, ${ }^{2}$ Catia Perciani, ${ }^{3}$ Thomas Murooka, ${ }^{4}$ Walter Jaoko, ${ }^{1}$ Kelly Macdonald, ${ }^{4}$ KAVI-ICR Team. ${ }^{1}$ University of Manitoba, Section of Infectious Diseases, Department of Internal Medicine, Winnipeg, Canada; ${ }^{2}$ University of Toronto, Department of Immunology, Toronto, Canada; ${ }^{3}$ University of Manitoba, Department of Immunology, Winnipeg, Canada; ${ }^{4}$ University of Nairobi, Kenya AIDS Vaccine Initiative (KAVI), Nairobi, Kenya

\subsection{6/sextrans-2019-sti.332}

Background Varicella-zoster virus (VZV), also known as chickenpox virus, constitutes a promising vector for a successful HIV vaccine. As an effort to scrutinize its potential, we are characterizing the susceptibility of VZV-specific CD4 T cells to HIV infection and the phenotypic profile of both CD4 and CD8 T cells.

Methods Blood T cells isolated from a cohort of healthy Kenyan women with pre-immunity to VZV (NCT02514018) were stimulated in vitro using 15 -mer peptides representing VZV glycoprotein E (gE) and VZV Open Reading Frame 4 (ORF4). CD4 and CD8 T cell memory phenotypes were characterized by flow cytometry based on the expression of CCR7/ CD45RA. The activation status of VZV-specific CD4 T cells was measured by the expression of HLA-DR, CD69, and CD25 after 6-day stimulation with gE and ORF4 peptides. Susceptibility to HIV infection was assessed using in vitro infection with a CCR5-tropic virus. DMSO and CMV peptides were used as negative and positive controls, respectively.

Results A similar frequency of central memory CD4 T cells $\left(\mathrm{T}_{\mathrm{CM}}\right)$ (median 24\%, IQR 18\%-32\%) and effector memory CD4 T cells ( $\left.\mathrm{T}_{\mathrm{EM}}\right)$ (median 27\%, IQR 20\%-32\%) was observed in our cohort. The predominant CD8 memory subtype was $\mathrm{T}_{\text {EMRA }}$ (median 28\%, IQR 21\%-40\%) followed by $\mathrm{T}_{\mathrm{EM}}$ cells (median 12\%, IQR 8\%-19\%) ( $\left.\mathrm{n}=45\right)$. Preliminary results show our ability to expand VZV-specific cells in culture using $\mathrm{gE}$ and ORF4 as stimuli and that these cells highly express the marker HLA-DR. Their susceptibility to in vitro $H I V$ infection is currently under investigation using CMV-specific cells as comparator.

Conclusion A viral vector able to sustain CD8 $\mathrm{T}_{\mathrm{EM}}$ responses without fueling the immune system with HIV target cells constitutes an ideal candidate for an HIV vaccine. Hence, our study sheds light on key aspects of VZV-specific immunity that will help determining its future as a vector in an HIV vaccine.

Disclosure No significant relationships. 